\title{
Resonant transmission of microwaves through subwavelength fractal slits in a metallic plate
}

\author{
Weijia Wen, Lei Zhou, Bo Hou, C. T. Chan, and Ping Sheng \\ Department of Physics, The Hong Kong University of Science and Technology, Clear Water Bay, Kowloon, Hong Kong, China
}

(Received 5 May 2005; revised manuscript received 12 August 2005; published 17 October 2005)

\begin{abstract}
We demonstrate through both experiment and theory that in the microwave regime one can have high transmittance through narrow fractal-patterned slits in thick metallic plates, whereby the aperture is subwavelength in all cross sectional dimensions. In contrast to the recently discovered extraordinary transmissions via surface plasmon excitations and Fabry-Perot-like resonances, the transmission in the present case is independent of the incident angle, plate thickness, or array periodicity. We show the physics to be governed by the transversal shape resonance localized in the metallic fractal slots. In particular, for the lowest transmission mode the EM field experiences no phase change when transmitting through the metallic plate. Simulation demonstrates the viability of the observed phenomenon as a subwavelength $k=0$ waveguide mode, where $k$ is the axial wave number.
\end{abstract}

DOI: 10.1103/PhysRevB.72.153406

PACS number(s): 73.20.Mf, 42.79.Dj, 41.20.Jb

Electromagnetic (EM) wave transmission through small metallic openings has been a topic of considerable fascination in recent years. Two mechanisms have been reported involving different types of resonances. In 1998, Ebbesen et al. showed that EM wave transmission through a silver film with a periodic array of subwavelength holes can be significantly higher than the conventional predictions, ${ }^{1-5}$ due to the excitation of surface plasmon (SP). Subsequently, Porto et $a l .{ }^{6}$ identified another waveguide mode resonance inside metallic slits due to the Febry-Perot (FP) interferences. ${ }^{7-9}$ In both cases the length scales relevant to the transmission mechanism must be comparable to the wavelength: in the SP case the periodicity must be comparable to the wavelength; the FP resonance requires at least one dimension of the slit cross section be comparable to the relevant wavelength, so that a fundamental TEM propagating wave mode ${ }^{10}$ may exist.

In this work, we demonstrate through both experiment and theory that in the microwave regime where SP is unlikely, one can have high transmittance through narrow slits in thick metallic plates arranged in a fractal geometry, in which the aperture cross section is subwavelength in all cross sectional dimensions. In contrast to the evanescent wave coupling mechanism, ${ }^{1}$ here the maximum transmission magnitude can range from $9 \%$ for a single fractal aperture to $100 \%$ for a $5 \times 5$ array of apertures. The high transmission is independent of periodicity of the array and incidence angle. In addition, the transmission frequency is also nearly independent of the sample thickness, in sharp contrast with the FP mechanism which depends strongly on the film thickness. ${ }^{6}$ We show the underlying physics to be governed by the localized resonance(s) of the fractal slit patterns.

The sample was prepared with stainless steel plates with different thicknesses on which a periodic array of fractal slits was generated by a diamond wire cutter. The unit cell of the array consists of a five-level structure, wherein the width of each slit is $0.8 \mathrm{~mm}$, with the longest slit being $1 \mathrm{~cm}$, as shown in Fig. 1. A total of 25 fractal slit units were made on a $12 \times 12 \mathrm{~cm}^{2}$ steel plate. This sample was mounted in the central window (with the same size as the $12 \times 12 \mathrm{~cm}^{2}$ ) of a $100 \times 100 \mathrm{~cm}$ steel plate so as to prevent microwave trans- mission through channels other than the fractal slit array. The microwave spectra were measured by a network analyzer (Agilent 8720ES). Two identical microwave horns (HP11966E) were used to generate and receive the signals separated by a distance of $100 \mathrm{~cm}$. The sample was placed on a stage, $15 \mathrm{~cm}$ from the receiving horn. The microwave polarization was such that the electric field is perpendicular to the shortest slits of the fractal pattern (defined as $E_{\perp}$, while $E_{\|}$is $90^{\circ}$ rotated). All measured spectra are normalized to the transmission when no sample is mounted. Transmission measurement for a single fractal slit aperture was carried out by covering all 24 apertures of the array with metallic sheets except the center one.

Figures 2(a) and 2(b) show the microwave transmission spectra through the slit array for two polarizations, where (a) and (b) are for $E_{\|}$and $E_{\perp}$, respectively. We note that for the thin sample, $\sim 100 \%$ transmittance can be identified at $5.1 \mathrm{GHz},>18 \mathrm{GHz}$ for the case of $E_{\|}$, and $3.0 \mathrm{GHz}$ and $10.0 \mathrm{GHz}$ for the case of $E_{\perp}$. The peak transmission frequency is downshifted somewhat when the thickness of stainless steel plate increases from $0.5 \mathrm{~mm}$ to $5.5 \mathrm{~mm}$, beyond which it stayed constant $(4.1 \mathrm{GHz}$ and $17.2 \mathrm{GHz}$ for $E_{\|}, 2.4 \mathrm{GHz}$ and $9.0 \mathrm{GHz}$ for $E_{\perp}$ ) up to the maximum sample thickness of $14.5 \mathrm{~mm}$. Transmission through a single fractal aperture was measured to be $9 \%$. In contrast, standard theory for a square hole with the same opening area $\left(99.5 \mathrm{~mm}^{2}\right)$ as the fractal aperture predicts transmission on the order of $<1 \%$. $^{5}$ It should be noted that at the lowest peak frequency,

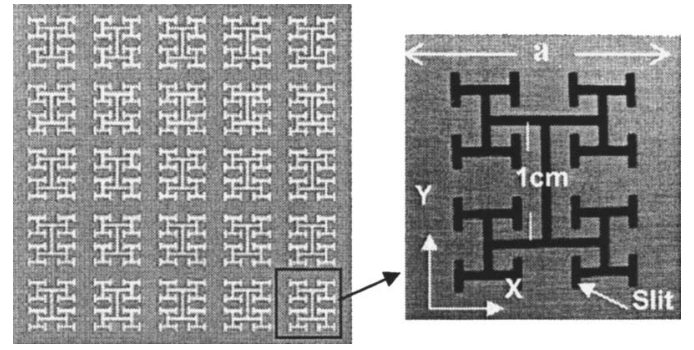

FIG. 1. A schematic picture of the fractal slit array and the unit cell. 

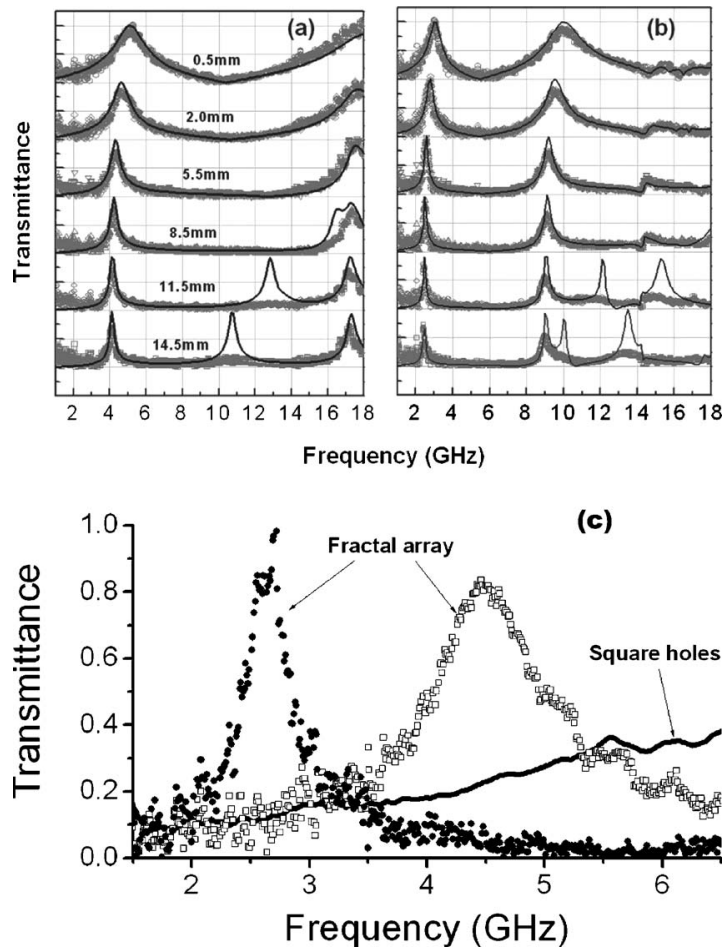

FIG. 2. Microwave transmittance through the $5 \times 5$ fractal arrays of various thicknesses for (a) $E_{\|}$polarization and (b) $E_{\perp}$ polarization; the thicknesses is denoted on the curves; the dark grey symbols and dark solid lines are the measured and simulated results, respectively; one vertical grid represents 50\% transmission. (c) Comparison of measured transmittances (of the two polarizations) for the fractal array are indicated as symbols on a $3 \mathrm{~mm}$-thick metallic plate with the square-hole array (solid dark line) on a metallic plate with the same $3 \mathrm{~mm}$ thickness. Fractal array and square holes have the same periodicity.

the incident wavelength $(12.5 \mathrm{~cm})$ is 12.5 times the longest slit $(1 \mathrm{~cm})$ on the steel plate. Hence the aperture cross section can be significantly subwavelength in both dimensions.

The transmission characteristics of the fractal slit array were investigated by finite difference time domain (FDTD) simulations. ${ }^{11,12}$ We consider an infinite plane tiled by a periodic replica of the five-level fractal slit patterns, and studied one unit cell with periodic conditions imposed at the outer boundaries. Perfect conductor boundary conditions, excellent for microwave frequencies, were applied to the metal/air interfaces. The simulation results are shown as white solid lines in Figs. 2(a) and 2(b). Except for the two thick samples, the agreement is seen to be excellent $(<1 \%$ difference). For the two thick samples some of the simulated transmission peaks are not seen experimentally, for reasons not yet clear. However, in all cases the lowest frequency, subwavelength transmission peak was always observed. The fact that the transmission peak persists to thin sample thicknesses points to localized fractal resonance(s) as the origin of this transmission mode. It was shown previously that planar metallic fractal patterns can have multiple localized resonances, and at the resonance frequencies the fractal plate can exhibit total reflection. ${ }^{13,14}$ Via Babinet's principle, ${ }^{15}$ the total reflection can become total transmission
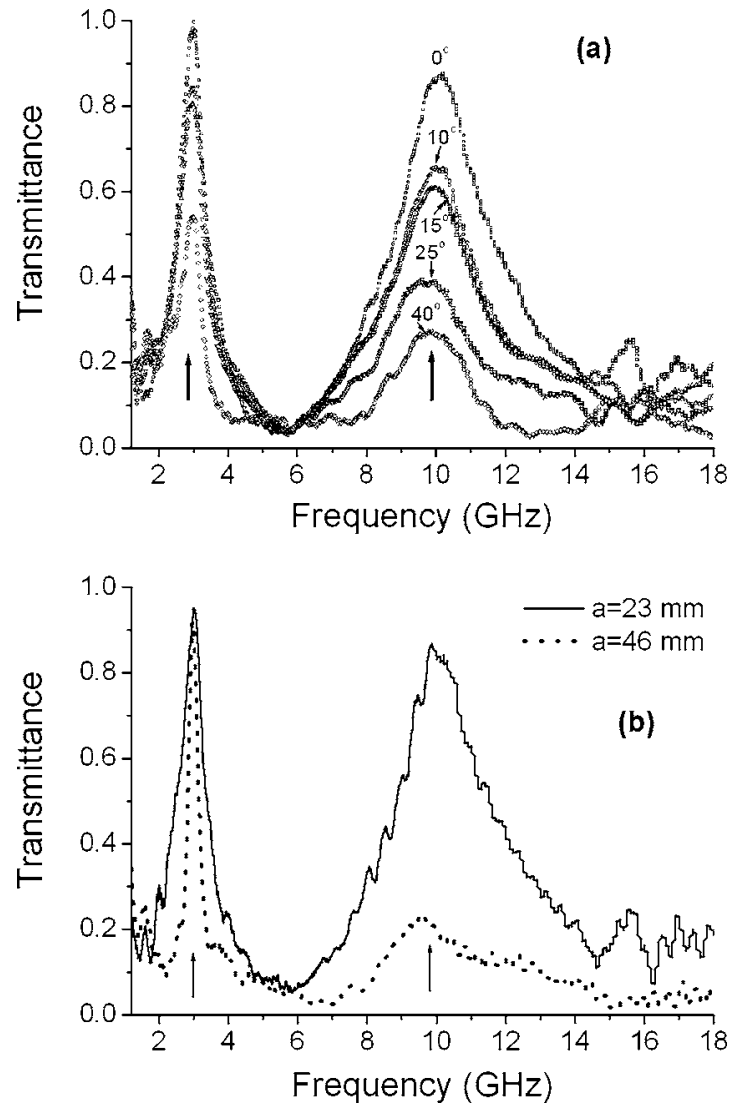

FIG. 3. Variation of the measured transmission spectra as a function of (a) the incident angle $\theta$ and (b) periodicity $a$. The sample is $0.5 \mathrm{~mm}$ thick and illuminated with $E_{\perp}$ polarization. The calculated peak frequencies are shown as arrows; they exhibit no variation with either the incident angle or the periodicity.

for the complimentary structure, accompanied by an interchange of electric and magnetic field configurations, plus a $90^{\circ}$ rotation of the pattern. The present transmission mode is simply a direct extension of such localized planar resonance(s). In particular, it can be regarded simply as the same resonance repeated at every slice of the sample. It is interesting to observe that for a reference sample consisting of an array of square holes $(18 \mathrm{~mm} \times 18 \mathrm{~mm}$ opening), the transmission is considerably lower, as shown in Fig. 2(c). Thus by removing metal, the sample becomes less transparent. The physics indicated here is that it is the contour line of the slit pattern (which can cause the resonances, see below) which matters, not the total size of the openings.

The periodicity and incident angle dependencies were also investigated, with experimental results shown in Fig. 3. Here the periodicity variation was achieved by covering 16 of the 25 apertures by metallic films, resulting effectively in a 3 by 3 array with twice the periodicity. It is seen that neither the periodicity of the array nor the incident angle of the EM wave has any noticeable effect on the positions of the transmission peaks, although the magnitude of the transmittance is affected to some degree. The simulation has confirmed this experimental observation, ${ }^{16}$ which is very different from the extraordinary optical transmission through twodimensional (2D) periodic array of holes caused by the 

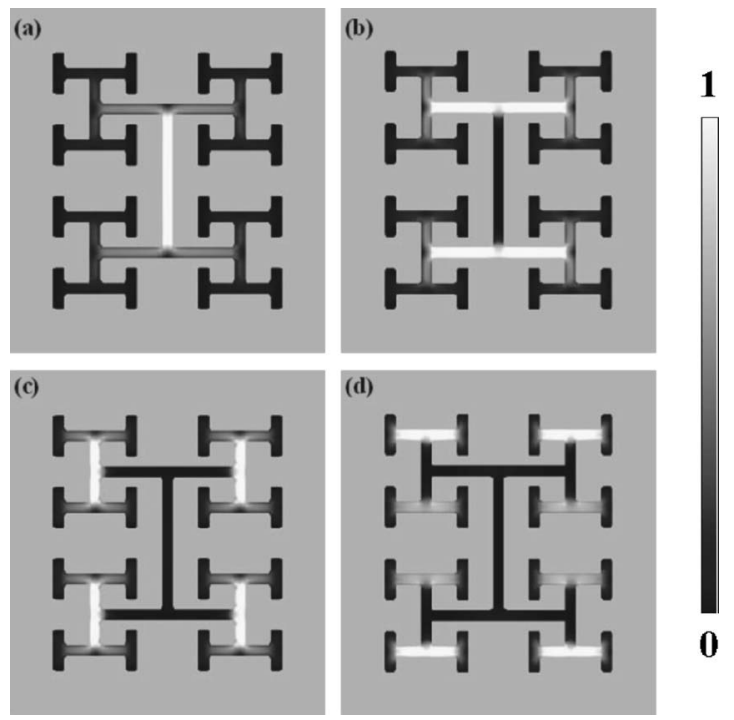

FIG. 4. The calculated field magnitude, $|E|$, inside the fractal slits at four frequencies: (a) $2.4 \mathrm{GHz}$, (b) $4.1 \mathrm{GHz}$, (c) $9.0 \mathrm{GHz}$, and (d) $17.2 \mathrm{GHz}$. In the grey scale, dark grey represents the strongest frequency. The sample thickness is taken to be $6 \mathrm{~mm}$.

excitation of SP resonance, where the transmission spectra are sensitive to the periodicity and strongly influenced by the incident angle. ${ }^{1-4}$ The insensitivity of the present transmission spectra to the plate thickness also distinguishes it from the FP resonances. ${ }^{6-9}$ In Figs. 2(a) and 2(b) there are also calculated FP modes (e.g., $10.7 \mathrm{GHz}$ and $12.8 \mathrm{GHz}$ in the $E_{\|}$ case). However, the measured transmissions are weaker than predicted. In fact, whereas the FP resonance mechanism works only when the plate is sufficiently thick, here the total transmission can persist to very thin thicknesses as seen from Fig. 2.

To obtain a physical picture of the subwavelength transmission mode, we calculated the $E$ field distributions inside the fractal slits at the four resonance frequencies: $2.4 \mathrm{GHz}$, 4.1 GHz, 9.0 GHz, and 17.2 GHz. The field in the slits forms a transverse-electric (TE) mode with significantly enhanced amplitude. The intensity distributions, shown in Fig. 4, illustrate these four resonant modes on the plane transverse to the propagation direction. It is seen that the different resonances are excited at the different levels of the fractal pattern. The lowest one $(2.4 \mathrm{GHz})$ has the highest intensity localized in the first level of the fractal pattern, while the next ones are localized at the higher levels of the fractal pattern, etc. The same behavior was seen in the complementary structure. ${ }^{13,16}$

In Fig. 5 we focus on the $2.4 \mathrm{GHz}$ transmission mode in the case of $6.0 \mathrm{~mm}$ plate thickness. From the $x y$-plane distribution pattern of the Poynting vector $S=E \times H^{*}$ (shown in Fig. 5(a) for an arbitrary transverse plane inside the sample), we find the field to be strongly enhanced inside the first-level fractal slit regions, but not confined just to the first level. We also find that the wave transmits across the metal mainly in the form of electric energy - while the $E$ field is strongly enhanced, there is no obvious enhancement in the $H$ field. Figure 5(b) shows the $E_{x}$ field pattern on the $y z$-plane in the central slit, where the field is approximately 23 times higher than the incident field. An important feature for the transmis-
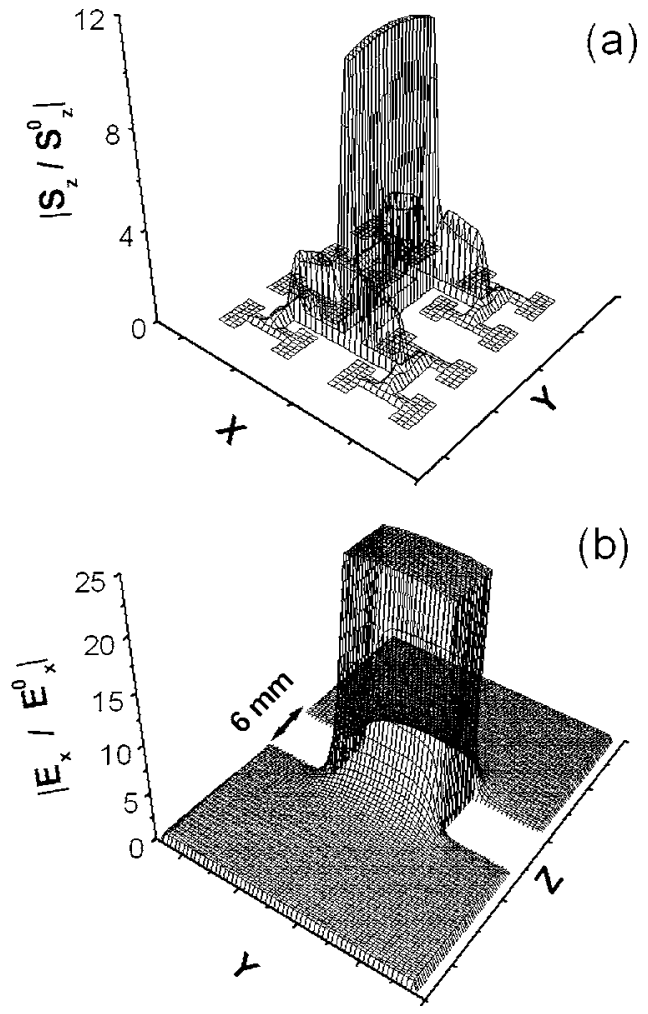

FIG. 5. (a) The calculated magnitude of the Poynting vector component $\left|S_{z}\right|$ for the $2.4 \mathrm{GHz}$ mode (normalized to the incident wave $\left|S_{z}^{0}\right|-E_{\perp}$-polarized with a frequency of $2.4 \mathrm{GHz}$ ) in an arbitrary $x y$-plane inside the metallic plate. (b) The $y z$-plane distribution of $\left|E_{x}\right|$ for the same mode [normalized to the same incident wave as (a)] inside the central slit. The sample thickness is taken to be $6 \mathrm{~mm}$.

sion process is that there is no phase change when an EM wave transmits across the metallic plate, that is to say, $k=0$ ( $k$ being the axial wave number). The localized nature of the resonance(s) also implies independence on the incidence angle and the periodicity.

The high transmission also implies very high coupling between the incident wave and the waveguide. This is some-

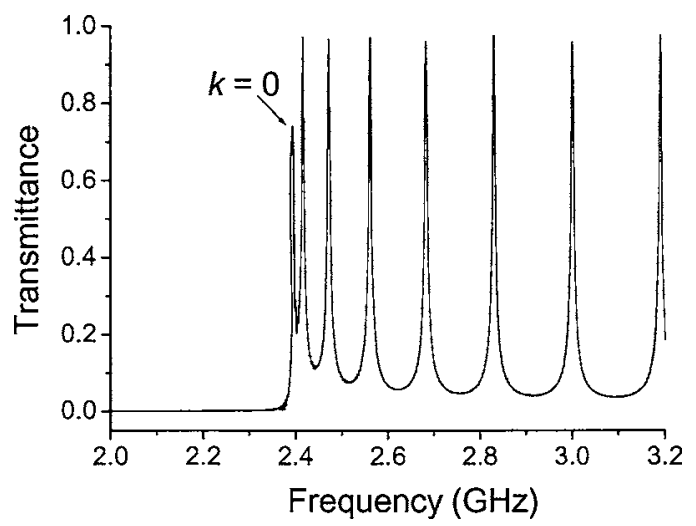

FIG. 6. Simulation result for a $5 \times 5$ fractal array with $50 \mathrm{~cm}$ thickness for the $E_{\perp}$ polarization. The $2.4 \mathrm{GHz}$ peak (the arrow indicates the $k=0$ peak) becomes extremely narrow, so that it cannot be resolved completely and thereby appears lower than $100 \%$. 
what puzzling at first sight because one expects a large impedance mismatch between the aperture and the air. Suppose the aperture is characterized by effective impedance $Z$ and a typical dispersion relation $k=\sqrt{\omega^{2}-\omega_{c}^{2}} / c$ in which $\omega_{c}$ is the cutoff frequency (or resonance frequency in our case). The transmission coefficient across such an aperture with thickness $W$ can be written down in analogy to transmission lines, i.e.,

$$
t=\frac{4 Z_{0} Z e^{i k W}}{\left(Z+Z_{0}\right)^{2}-\left(Z-Z_{0}\right)^{2} e^{i 2 k W}},
$$

where $Z_{0}$ is the impedance for air. When the impedance contrast is high $\left(Z \ll Z_{0}\right.$ or $\left.Z \gg Z_{0}\right)$, it is easy to check that $t \sim 0$. However, Eq. (1) predicts perfect transmission $(t=1)$ for $k \rightarrow 0$. The latter implies sample thickness independence. It should be noted that Eq. (1) was derived originally for nonzero $k$, finite $Z$ transmission lines, and the above conclusion (i.e., $t=1$ for $k \rightarrow 0$ ) holds for every frequency component.
Hence even if $Z$ is frequency dependent or the interface between the structure and the air is reactive, $t=1$ still holds provided $k \rightarrow 0$.

In Fig. 6 we show the simulation result for the $5 \times 5$ array of fractal slits that are $50 \mathrm{~cm}$ in thickness. In the simulation, periodic boundary condition and perfect conductor approximation were assumed just as above. It is seen that increased thickness means many more FP transmission peaks. However, the $2.4 \mathrm{GHz}$ transmission peak is preserved with a narrower width. As the thickness is now many wavelengths, the lowest frequency transmission may be viewed as a $k=0$ waveguide mode.

In summary, we show that by using fractal cross sectional geometry, subwavelength total transmission through metallic slits can be achieved. The effect is robust and should be applicable to infrared frequencies as well.

This work is supported by Hong Kong RGC through CA02/03.SC01.
${ }^{1}$ T. W. Ebbesen, H. J. Lezec, H. F. Ghaemi, T. Thio, and P. A. Wolff, Nature (London) 391, 667 (1998).

${ }^{2}$ H. F. Ghaemi, T. Thio, D. E. Grupp, T. W. Ebbesen, and H. J. Lezec, Phys. Rev. B 58, 6779 (1998).

${ }^{3}$ L. Martín-Moreno, F. J. García-Vidal, H. J. Lezec, K. M. Pellerin, T. Thio, J. B. Pendry, and T. W. Ebbesen, Phys. Rev. Lett. 86, 1114 (2001).

${ }^{4}$ W. L. Barnes, W. A. Murray, J. Dintinger, E. Devaux, and T. W. Ebbesen, Phys. Rev. Lett. 92, 107401 (2004).

${ }^{5}$ H. A. Bethe, Phys. Rev. 66, 163 (1944).

${ }^{6}$ J. A. Porto, F. J. Garcia-Vidal, and J. B. Pendry, Phys. Rev. Lett. 83, 2845 (1999).

${ }^{7}$ Y. Takakura, Phys. Rev. Lett. 86, 5601 (2001).

${ }^{8}$ F. Yang and J. R. Sambles, Phys. Rev. Lett. 89, 063901 (2002).

${ }^{9}$ H. E. Went, A. P. Hibbins, J. R. Sambles, C. R. Lawrence, and A.
P. Crick, Appl. Phys. Lett. 77, 2789 (2000).

${ }^{10}$ E. Popov, M. Nevière, S. Enoch, and R. Reinisch, Phys. Rev. B 62, 16100 (2000).

${ }^{11}$ K. S. Yee, IEEE Trans. Antennas Propag. 14, 302 (1966).

${ }^{12}$ Simulations were performed using the package CONCERTO 3.1, developed by Vector Fields Limited, England, 2002.

${ }^{13}$ W. Wen, L. Zhou, J. Li, W. Ge, C. T. Chan, and P. Sheng, Phys. Rev. Lett. 89, 223901 (2002).

${ }^{14}$ L. Zhou, W. Wen, C. T. Chan, and P. Sheng, Appl. Phys. Lett. 82, 1012 (2003).

${ }^{15}$ B. A. Munk, Frequency Selective Surfaces, Theory and Design (Wiley, New York, 2000).

${ }^{16}$ The calculation of transmission under off-normal incidence follows the method described in L. Zhou, C. T. Chan, and P. Sheng, J. Phys. D 37, 368 (2004). 